\title{
BEHAVIOUR OF SUPERIOR GRAPEVINES TO APPLICATION OF SOME REST BREAKING AGENTS AND WINTER PRUNING II- EFFECT OF TIMES OF WINTER PRUNING AND DIFFERENT VINE LOADS
}

Faissal F. Ahmed ; Moawad M. Abd El-Hameed ${ }^{*}$; Mervat A. Aly** andAhmed Y. El-Saman ${ }^{* * *}$

*Hort. Dept. Fac. of Agric. Minia Univ. Egypt

***Viticulture Res. Hort. Res. Instit. ARC, Giza, Egypt

E mail: faissalfadel@yahoo.com

\section{ABSTRACT}

This study was conducted during 2011/2012 and 2012/2013 seasons to adjust the best date of winter pruning ( $24^{\text {th }}$ Dec., $31^{\text {st }}$ Dec. or $7^{\text {th }}$ Jan. $)$ as well as vine load levels $(66,72,78$ or 84 eyes/vine) that responsible for gaining higher yield with fairly good berries quality of Superior grapevine cv.

Delaying dates of winter pruning from $24^{\text {th }}$ Dec. to $7^{\text {th }}$ Jan. considerably enhanced the perentages of bud burst and fruiting buds, yield, shot berries\%, T.S.S.\%, and reducing sugars $\%$ and reduced leaf area, wood ripening coefficient, total chlorophylls and N, P, K, berry weight and total acidity\%. All the investigated characteristics except bud burst $\%$, berry weight and total acidity\% were gradually increased with increasing vine loads from 66 to 84 eyes/vine.

Pruning on $7^{\text {th }}$ Jan. leaving 84 eyes/vine (6 fruiting cane $x 12$ eyes plus 6 renewal spurs $x$ two eyes) gave the best results with regard to productivity of Superior grapevine cv.

Keywords: Dates of pruning, Vine load, bud burst, Fruiting buds, growth, yield, berries quality, Superior grapevines.

\section{INTRODUCTION}

Pruning is the most important and vital cultural practice in the management of different grapevine cvs. It is a limiting factor for producing an economical yield and better quality of berries (Chapman, 1990). It is done to select the suitable vine load and maintain vine shape. It must be done each dormant season and it directly influences yield, fruit quality, vine vigour and hardiness. Proper pruning with the optimum vine load and proper date will result in improving yield quantitively and qualitatively (Akopyan and Khasapetyan, 1994).

Improper date of winter pruning (Al-Khayat and Al-Dujaili, 2001), vine loads and fruiting canes length were accompanied with unfavourable impact on yield and berries characteristics in different grapevine cvs. (Attia, 1998; Avenant, 1998; Ahmed-Ansam, 2002; Jarad, 2004;Hussein, 2009; Fawzi et al., 2010; Abdel- Mohsen, 2013; Abu- Zinada, 2015; Aly et al., 2015; Rahmani et al., 2015 and Khalaf, 2017 ). 
Faissal F. Ahmed"; et al.,

The target of this was examining the effect of dates of winter pruning and different levels of vine loads on behavior of buds, growth, yield and berries quality of Superior grapevines grown under Fayoum environmental conditions.

\section{MATERIALS AND METHODS}

This study was carried out during the two consecutive seasons of 2011/2012 and 2012/2013 on seventy-two uniform in vigour 8-years old Superior grapevines grown in a private vineyard located at Ahmed Afendy Village, Youssef El-Sediek district, Fayoum Governorate where the soil texture is clay and well drained water since water table depth is not less than two meters. The chosen vines are planted at $2 \times 3$ meters apart. Generally, Cane pruning system was practiced at the first week of January. leaving different vine loads according to the present treatments with the assistance of Baron shape supporting system. The vines were irrigated through surface irrigation system using Nile water. The selected vines received the same and common horticultural practices that already applied in the vineyard except pruning treatments.

This study contained two factors (A x B).

The first factor (A) compressed from three dates of winter pruning namely

$\mathrm{a}_{1}$ ) Carrying out pruning on Dec 24.

$\mathrm{a}_{2}$ ) Carrying out pruning on Dec31.

$\mathrm{a}_{3}$ ) Carrying out pruning on Jan 7.

The second factor (B) included four vine loads and fruiting spurs lengths namely:

b1) leaving six fruiting spurs each with nine eyes plus six renewal spurs each with two eyes with a total vine load of 66 eyes.

$b_{2}$ ) leaving six fruiting spurs each with ten eyes plus six renewal spurs each with two eyes with a total vine load of 72 eyes.

$b_{3}$ ) leaving six fruiting spurs each with eleven eyes plus six renewal spurs each with two eyes with a total vine load of 78 eyes.

$b_{4}$ ) leaving six fruiting spurs each with twelve eyes plus six renewal spurs each with two eyes with a total vine load of 84 eyes.

Each treatment was replicated three times, two vines per each.

Randomized complete block design (RCBD) in splite plote arrangement was adopted where the three dates of pruning occupied the whole plots and the four different vine loads ranked the sub-plots.

During both seasons, the following parameters were recorded:

1 - Percentages of bud burst and fruiting buds.

2- Vegetative growth characteristics namely leaf area (Ahmed and Morsy, 1999) and wood ripening coefficient (Bouard, 1966).

3 - Percentages of N, P and $\mathrm{K}$ in the leaf petioles (on dry weight basis) (according to Wilde et al., 1985 and Summer, 1985).

4- Total chlorophylls (chlorophyll a \& b) (Von-Wettstein, 1957) (mg/100g F.W).

5- Percentage of berry setting.

6- Harvesting date.

7- Yield/vine expressed in weight (kg.) and number of clusters/vine.

Fayoum J. Agric. Res. \& Dev., Vol. 31, No.2, July, 2017 
8- Cluster weight (g.).

9- Percentage of shot berries.

10- Physical and chemical characteristics of the berries namely berry weight and total soluble solids $\%$, total acidity $\%$ expressed as $\mathrm{g} / \mathrm{ml}$ juice tartaric acid and reducing sugars\% (Lane and Eynon, 1985) (A.O.A.C., 2000).

The obtained data was tabulated and subjected to the proper statistical analysis using new L.S.D. at 5\% (Mead et al., 1993).

\section{RESULTS AND DISCUSSION}

\section{Percentages of bud burst and fruiting buds:}

Data in Table (1) clearly show that delaying dates of pruning from $24^{\text {th }}$ Dec. to $7^{\text {th }}$ Jan. was significantly followed by a gradual promotion on the percentages of bud burst and fruiting buds. Percentage of bud burst was significantly reduced gradually, while percentage of fruiting buds was significantly promoted gradually with increasing vine loads from 66 to 84 eyes/vine and fruiting canes lengths from 9 to 12 eyes per each. Similar trend was noticed during both seasons.

\section{Growth as well as total chlorophylls, $N, P$ and $K$ in the leaves:}

Data in Tables $(1 \& 2)$ obviously clear that delaying dates of pruning from $24^{\text {th }}$ Dec. to $7^{\text {th }}$ Jan. significantly caused a gradual promotion on the leaf area and reduction on wood ripening coefficient, total chlorophylls, $\mathrm{N}, \mathrm{P}$ and $\mathrm{K}$. Increasing vine loads from 66 to 84 eyes/vine and lengths of fruiting canes from 9 to 12 eyes per each was significantly followed by a gradual promotion on the leaf area, wood ripening coefficient, total chlorophylls, $\mathrm{N}, \mathrm{P}$ and $\mathrm{K}$. These results were true during both seasons.

Table (1): Effect of different dates of pruning and/or vine load levels on the percentages of bud burst, fruiting buds, leaf area and wood ripening coefficient of Superior grapevines during 2011/2012 and 2012/2013 seasons

\begin{tabular}{|c|c|c|c|c|c|c|c|c|c|c|c|c|c|c|c|c|}
\hline \multirow{4}{*}{$\begin{array}{l}\text { Vine load } \\
\text { levels ( B ) }\end{array}$} & \multicolumn{8}{|c|}{ Bud burst \% } & \multicolumn{8}{|c|}{ Fruiting buds \% } \\
\hline & \multicolumn{4}{|c|}{ 2011/2012 } & \multicolumn{4}{|c|}{$2012 / 2013$} & \multicolumn{4}{|c|}{$2011 / 2012$} & \multicolumn{4}{|c|}{$2012 / 2013$} \\
\hline & \multicolumn{16}{|c|}{ Date of pruning (A) } \\
\hline & $\begin{array}{c}\mathrm{a}_{1} \\
24 \\
\text { Dec. }\end{array}$ & $\begin{array}{c}\mathbf{a}_{2} \\
31 \\
\text { Dec. }\end{array}$ & $\begin{array}{c}\mathbf{a}_{3} \\
7 \\
\text { Jan. }\end{array}$ & $\begin{array}{c}\text { Mean } \\
\text { ( B ) }\end{array}$ & $\begin{array}{c}\mathbf{a}_{1} \\
24 \\
\text { Dec. }\end{array}$ & $\begin{array}{l}\mathbf{a}_{2} \\
\mathbf{3 1} \\
\text { ec. }\end{array}$ & $\begin{array}{c}a_{3} \\
7 \\
\text { Jan. }\end{array}$ & $\begin{array}{c}\text { Mean } \\
\text { ( B ) }\end{array}$ & $\begin{array}{c}\mathbf{a}_{1} \\
\mathbf{2 4} \\
\text { Dec. }\end{array}$ & $\begin{array}{c}\mathbf{a}_{2} \\
31 \\
\text { Dec. }\end{array}$ & $\begin{array}{c}\mathbf{a}_{3} \\
7 \\
\text { Jan. }\end{array}$ & $\begin{array}{c}\text { Mean } \\
\text { ( B ) }\end{array}$ & $\begin{array}{c}a_{1} \\
24 \\
\text { Dec. }\end{array}$ & $\begin{array}{c}\mathbf{a}_{2} \\
\mathbf{3 1} \\
\text { Dec. }\end{array}$ & $\begin{array}{c}\mathbf{a}_{3} \\
7 \\
\text { Jan. }\end{array}$ & $\begin{array}{c}\text { Mean } \\
\text { ( B ) }\end{array}$ \\
\hline b1 66 eyes/vine & 78.1 & 80.2 & 82.0 & 80.1 & 79.4 & 81.5 & 83.6 & 81.5 & 41.1 & 43.3 & 46.0 & 43.5 & 43.0 & 45.3 & 48.9 & 45.7 \\
\hline b2 72 eyes/vine & 76.0 & 78.0 & 80.0 & 78.0 & 77.0 & 79.0 & 81.2 & 79.1 & 43.3 & 45.9 & 48.9 & 46.0 & 45.5 & 48.0 & 51.0 & 48.2 \\
\hline b3 78 eyes/vine & 74.0 & 76.0 & 78.0 & 76.0 & 75.0 & 77.0 & 79.0 & 77.1 & 45.9 & 48.9 & 51.9 & 48.9 & 48.0 & 51.2 & 54.0 & 51.1 \\
\hline b4 84 eyes/vine & 72.0 & 74.0 & 76.3 & 74.1 & 73.0 & 75.0 & 77.0 & 75.0 & 48.0 & 52.0 & 54.0 & 51.3 & 50.0 & 53.9 & 56.0 & 53.3 \\
\hline Mean (A) & 75.0 & 77.1 & 79.1 & & 76.1 & 78.1 & 80.2 & & 44.6 & 47.5 & 50.2 & & 46.6 & 49.6 & 52.5 & \\
\hline New L.S.D at 5\% & $\begin{array}{c}A \\
1.6\end{array}$ & $\begin{array}{c}\text { B } \\
1.8\end{array}$ & $\begin{array}{l}\text { AB } \\
3.1\end{array}$ & & $\begin{array}{c}\text { A } \\
1.9\end{array}$ & $\begin{array}{c}\text { B } \\
2.0\end{array}$ & $\begin{array}{l}\text { AB } \\
3.5\end{array}$ & & $\begin{array}{c}A \\
1.0\end{array}$ & $\begin{array}{c}\text { B } \\
1.0\end{array}$ & $\begin{array}{c}\text { AB } \\
1.7\end{array}$ & & $\begin{array}{c}A \\
1.0\end{array}$ & $\begin{array}{c}\text { B } \\
1.0\end{array}$ & $\begin{array}{l}\text { AB } \\
1.7\end{array}$ & \\
\hline Charac & \multicolumn{8}{|c|}{ Leaf area $(\mathrm{cm})^{\frac{1}{2}}$} & \multicolumn{8}{|c|}{ Wood ripening coefficient } \\
\hline b1 66 eyes/vine & 106.0 & 104.0 & 102.0 & 104.0 & 105.9 & 103.8 & 101.0 & 103.6 & 0.79 & 0.73 & 0.67 & 0.73 & 0.80 & 0.79 & 0.72 & 0.77 \\
\hline b2 72 eyes/vine & 109.0 & 107.0 & 104.9 & 107.0 & 110.0 & 106.7 & 103.0 & 106.6 & 0.87 & 0.8 & 0.73 & 0.80 & 0.87 & 0.80 & 0.73 & 0.80 \\
\hline b3 78 eyes/vine & 111.0 & 109.0 & 106.9 & \begin{tabular}{|l|}
109.0 \\
\end{tabular} & 113.0 & 110.0 & 106.8 & 109.9 & 0.91 & 0.83 & 0.77 & 0.84 & 0.94 & 0.87 & 0.8 & 0.87 \\
\hline b4 84 eyes/vine & 113.0 & 110.9 & 108.0 & 110.6 & 111.9 & 113.0 & 109.9 & 111.6 & 0.95 & 0.89 & 0.83 & 0.89 & 0.97 & 0.90 & 0.83 & 0.90 \\
\hline $\operatorname{Mean}($ A ) & 109.8 & 107.7 & 105.5 & & 110.2 & 108.4 & 105.2 & & 0.88 & 0.81 & 0.75 & & 0.90 & 0.84 & 0.77 & \\
\hline New L.S.D at $5 \%$ & $\begin{array}{c}A \\
1.2\end{array}$ & $\begin{array}{l}B \\
1.3\end{array}$ & $\begin{array}{l}\mathbf{A B} \\
2.2\end{array}$ & & $\begin{array}{c}A \\
1.3\end{array}$ & $\begin{array}{c}\text { B } \\
1.4\end{array}$ & $\begin{array}{l}\mathrm{AB} \\
2.4\end{array}$ & & $\begin{array}{c}\text { A } \\
0.07\end{array}$ & $\begin{array}{c}\text { B } \\
0.06\end{array}$ & $\begin{array}{c}\text { AB } \\
0.10\end{array}$ & & $\begin{array}{c}\text { A } \\
0.07\end{array}$ & $\begin{array}{c}\text { B } \\
0.07\end{array}$ & $\begin{array}{c}\text { AB } \\
0.11\end{array}$ & \\
\hline
\end{tabular}

Fayoum J. Agric. Res. \& Dev., Vol. 31, No.2, July, 2017 
Faissal F. Ahmed ; ; et al.,

Table (2): Effect of different dates of pruning and/or vine load levels on total chlorophylls and percentages of $\mathrm{N}, \mathrm{P}$ and $\mathrm{K}$ in the leaves of Superior grapevines during 2011/2012 and 2012/2013 seasons.

\begin{tabular}{|c|c|c|c|c|c|c|c|c|c|c|c|c|c|c|c|c|}
\hline \multirow{4}{*}{$\begin{array}{c}\text { Vine load } \\
\text { levels ( B ) }\end{array}$} & \multicolumn{8}{|c|}{ Total chlorophylls (mg/100 g F.W) } & \multicolumn{8}{|c|}{ Leaf N \% } \\
\hline & \multicolumn{4}{|c|}{ 2011/2012 } & \multicolumn{4}{|c|}{$2012 / 2013$} & \multicolumn{4}{|c|}{$2011 / 2012$} & \multicolumn{4}{|c|}{$2012 / 2013$} \\
\hline & \multicolumn{16}{|c|}{ Date of pruning (A) } \\
\hline & \begin{tabular}{|c}
$a_{1}$ \\
24 \\
Dec. \\
\end{tabular} & $\begin{array}{r}\mathbf{a}_{2} \\
31 \\
\text { Dec. }\end{array}$ & $\begin{array}{c}\mathbf{a}_{3} \\
7 \\
\text { Jan. }\end{array}$ & $\begin{array}{l}\text { Mean } \\
\text { ( B ) }\end{array}$ & \begin{tabular}{|c}
$a_{1}$ \\
24 \\
Dec.
\end{tabular} & $\begin{array}{c}\mathbf{a}_{2} \\
\mathbf{3 1} \\
\text { Dec. }\end{array}$ & $\begin{array}{c}\mathrm{a}_{3} \\
7 \\
\text { Jan. } \\
\end{array}$ & $\begin{array}{c}\text { Mean } \\
\text { ( B ) }\end{array}$ & $\begin{array}{c}\mathbf{a}_{1} \\
24 \\
\text { Dec. }\end{array}$ & $\begin{array}{r}\mathbf{a}_{2} \\
\mathbf{3 1} \\
\text { Dec } \\
\end{array}$ & $\begin{array}{c}\mathbf{a}_{3} \\
7 \\
\text { Jan. }\end{array}$ & $\begin{array}{c}\text { Mean } \\
\text { ( B ) }\end{array}$ & $\begin{array}{c}\mathbf{a}_{1} \\
24 \\
\text { Dec. }\end{array}$ & $\begin{array}{r}\mathbf{a}_{2} \\
\mathbf{3 1} \\
\text { Dec. }\end{array}$ & $\begin{array}{c}\mathbf{a}_{3} \\
7 \\
\text { Jan. }\end{array}$ & $\begin{array}{c}\text { Mean } \\
\text { ( B ) }\end{array}$ \\
\hline b1 66 eyes/vine & 6.5 & 5.6 & 4.8 & 5.6 & 7.1 & 6.3 & 5.3 & 6.2 & 1.59 & 1.53 & 1.46 & 1.53 & 1.46 & 1.54 & 1.44 & 1.48 \\
\hline b2 72 eyes/vine & 7.4 & 6.5 & 5.5 & 6.5 & 8.0 & 6.9 & 5.9 & 6.9 & 1.66 & 1.57 & 1.50 & 1.58 & 1.80 & 1.63 & 1.53 & 1.65 \\
\hline b3 78 eyes/vine & 8.2 & 7.2 & 6.3 & 7.2 & 9.0 & 8.0 & 7.1 & 8.0 & 1.73 & 1.66 & 1.59 & 1.66 & 1.90 & 1.8 & 1.63 & 1.78 \\
\hline b4 84 eyes/vine & 9.1 & 8 & 6.9 & 8.3 & 10.0 & 8.7 & 7.9 & 8.9 & 1.81 & 1.72 & 1.66 & 1.73 & 1.96 & 1.89 & 1.79 & 1.88 \\
\hline Mean (A) & 7.8 & 6.8 & 6.1 & & 8.5 & 7.5 & 6.6 & & 1.70 & 1.62 & 1.55 & & 1.78 & 1.72 & 1.60 & \\
\hline New L.S.D at $5 \%$ & $\begin{array}{c}\text { A } \\
0.4 \\
\end{array}$ & $\begin{array}{c}\text { B } \\
0.4\end{array}$ & $\begin{array}{l}\text { AB } \\
0.7 \\
\end{array}$ & & \begin{tabular}{|c|} 
A \\
0.4 \\
\end{tabular} & $\begin{array}{c}\text { B } \\
0.4\end{array}$ & $\begin{array}{l}\text { AB } \\
\text { 0.7 }\end{array}$ & & $\begin{array}{c}\text { A } \\
0.06 \\
\end{array}$ & \begin{tabular}{|c|} 
B \\
0.07 \\
\end{tabular} & $\begin{array}{c}\text { AB } \\
0.12 \\
\end{array}$ & & $\begin{array}{c}\text { A } \\
0.06 \\
\end{array}$ & $\begin{array}{c}\text { B } \\
0.07 \\
\end{array}$ & $\begin{array}{c}\text { AB } \\
0.12 \\
\end{array}$ & \\
\hline Characteristics & \multicolumn{8}{|c|}{ Leaf P \% } & \multicolumn{8}{|c|}{ Leaf K \% } \\
\hline b1 66 eyes/vine & 0.12 & 0.10 & 0.08 & 0.10 & 0.11 & 0.09 & 0.07 & \begin{tabular}{|l|}
0.10 \\
\end{tabular} & 1.39 & 1.3 & 1.19 & 1.29 & 1.40 & 1.30 & 1.20 & 1.30 \\
\hline b2 72 eyes/vine & 0.15 & 0.11 & 0.09 & 0.12 & 0.13 & 0.11 & 0.09 & \begin{tabular}{|l|}
0.11 \\
\end{tabular} & 1.46 & 1.38 & 1.30 & 1.38 & 1.50 & 1.39 & 1.30 & 1.40 \\
\hline b3 78 eyes/vine & 0.17 & 0.14 & 0.12 & 0.14 & 0.16 & 0.13 & 0.11 & 0.13 & 1.53 & 1.45 & 1.35 & 1.44 & 1.61 & 1.49 & 1.41 & 1.50 \\
\hline b4 84 eyes/vine & 0.20 & 0.17 & 0.14 & 0.17 & 0.18 & 0.15 & 0.13 & \begin{tabular}{|l|}
0.15 \\
\end{tabular} & 1.60 & 1.52 & 1.41 & 1.51 & 1.71 & 1.60 & 1.51 & 1.61 \\
\hline Mean (A) & 0.16 & 0.13 & 0.11 & & 0.15 & 0.12 & 0.10 & & 1.50 & 1.41 & 1.31 & & 1.56 & 1.45 & 1.36 & \\
\hline New L.S.D at $5 \%$ & $\begin{array}{c}\mathrm{A} \\
\mathbf{0 . 0 2}\end{array}$ & $\begin{array}{c}\text { B } \\
0.02\end{array}$ & $\begin{array}{c}\text { AB } \\
\text { 0.03 }\end{array}$ & & \begin{tabular}{|c|} 
A \\
0.02
\end{tabular} & $\begin{array}{c}\text { B } \\
0.02\end{array}$ & \begin{tabular}{|c|} 
AB \\
0.03
\end{tabular} & & $\begin{array}{c}A \\
0.05\end{array}$ & \begin{tabular}{|c|} 
B \\
0.05
\end{tabular} & $\begin{array}{c}\text { AB } \\
0.09\end{array}$ & & $\begin{array}{c}\text { A } \\
0.04\end{array}$ & $\begin{array}{c}\text { B } \\
0.05\end{array}$ & $\begin{array}{c}\text { AB } \\
0.09\end{array}$ & \\
\hline
\end{tabular}

\section{Yield/vine and cluster weight:}

Data in Table (3) noticeably revealed that yield/vine and cluster weight were significantly improved in response to pruning on $31^{\text {th }}$ Dec. and $7^{\text {th }}$ Jan. than pruning on $24^{\text {th }}$ Dec. There was significant and gradual promotion on the yield and cluster weight with delaying dates of pruning. Increasing vine loads from 66 to 84 eyes/vine and fruiting canes lengths from 9 to 12 eyes per each caused a significant and gradual promotion on the yield and cluster weight. Similar trend was noticed during both seasons.

Table (3): Effect of different dates of pruning and/or vine load levels on the number of clusters/vine, yield/vine, cluster weight (g.) and shot berries of Superior grapevines during 2011/2012 and 2012/2013 seasons.

\begin{tabular}{|c|c|c|c|c|c|c|c|c|c|c|c|c|c|c|c|c|}
\hline \multirow{4}{*}{$\begin{array}{l}\text { Vine load } \\
\text { levels ( B ) }\end{array}$} & \multicolumn{8}{|c|}{ No. of clusters/vine } & \multicolumn{8}{|c|}{ Yield/vine (kg.) } \\
\hline & \multicolumn{4}{|c|}{$2011 / 2012$} & \multicolumn{4}{|c|}{$2012 / 2013$} & \multicolumn{4}{|c|}{ 2011/2012 } & \multicolumn{4}{|c|}{$2012 / 2013$} \\
\hline & \multicolumn{16}{|c|}{ Date of pruning (A) } \\
\hline & $\begin{array}{c}a_{1} \\
24 \\
\text { Dec. }\end{array}$ & $\begin{array}{c}\mathbf{a}_{2} \\
31 \\
\text { Dec. }\end{array}$ & $\begin{array}{c}\mathbf{a}_{3} \\
7 \\
\text { Jan. }\end{array}$ & $\begin{array}{c}\text { Mean } \\
(\text { B })\end{array}$ & $\begin{array}{c}a_{1} \\
24 \\
\text { Dec. }\end{array}$ & $\begin{array}{c}\mathbf{a}_{2} \\
31 \\
\text { Dec. } \\
\end{array}$ & \begin{tabular}{|c|}
$\mathbf{a}_{3}$ \\
7 \\
Jan. \\
\end{tabular} & $\begin{array}{c}\text { Mean } \\
\text { ( B ) }\end{array}$ & $\begin{array}{c}a_{1} \\
24 \\
\text { Dec. }\end{array}$ & $\begin{array}{c}\mathbf{a}_{2} \\
31 \\
\text { Dec. }\end{array}$ & $\begin{array}{c}\mathbf{a}_{3} \\
7 \\
\text { Jan. }\end{array}$ & $\begin{array}{c}\text { Mean } \\
(\text { B })\end{array}$ & \begin{tabular}{|c|}
$a_{1}$ \\
24 \\
Dec.
\end{tabular} & $\begin{array}{c}\mathbf{a}_{2} \\
31 \\
\text { Dec. }\end{array}$ & $\begin{array}{c}a_{3} \\
7 \\
\text { Jan. }\end{array}$ & $\begin{array}{l}\text { Mean } \\
\text { ( B ) }\end{array}$ \\
\hline b1 66 eyes/vine & 20.0 & 20.0 & 20.0 & 20.0 & 20.0 & 22.0 & 24.0 & 22.0 & 7.0 & 7.2 & 7.4 & 7.2 & 6.8 & 7.8 & 8.7 & 7.8 \\
\hline b2 72 eyes/vine & 20.0 & 20.0 & 20.0 & 20.0 & 23.0 & 25.0 & 27.0 & 25.0 & 7.2 & 7.4 & 7.6 & 7.4 & 8.1 & 9.1 & 10.1 & 9.1 \\
\hline b3 78 eyes/vine & 21.0 & 21.0 & 21.0 & 21.0 & 25.0 & 27.0 & 29.0 & 27.0 & 7.8 & 8.0 & 8.3 & 8.0 & 9.1 & 10.1 & 11.1 & 10.1 \\
\hline b4 84 eyes/vine & 21.0 & 21.0 & 21.0 & 21.0 & 28.0 & 32.0 & 32.0 & 30.7 & 7.8 & 8.4 & 8.6 & 8.3 & 10.5 & 12.4 & 12.7 & 11.9 \\
\hline Mean ( A ) & 20.5 & 20.5 & 20.5 & & 24.0 & 26.5 & 28.0 & & 7.5 & 7.8 & 8.0 & & 8.6 & 9.9 & 10.7 & \\
\hline New L.S.D at 5\% & $\begin{array}{c}\mathrm{A} \\
\mathrm{NS}\end{array}$ & $\begin{array}{c}\text { B } \\
\text { NS }\end{array}$ & $\begin{array}{l}\text { AB } \\
\text { NS }\end{array}$ & & $\begin{array}{c}\text { A } \\
2.0\end{array}$ & $\begin{array}{c}B \\
2.0\end{array}$ & \begin{tabular}{l|}
$\mathrm{AB}$ \\
3.5
\end{tabular} & & $\begin{array}{c}\mathrm{A} \\
0.2\end{array}$ & $\begin{array}{c}\text { B } \\
0.3\end{array}$ & $\begin{array}{c}\text { AB } \\
0.5\end{array}$ & & $\begin{array}{c}\mathrm{A} \\
0.4\end{array}$ & $\begin{array}{c}\text { B } \\
0.5\end{array}$ & $\begin{array}{l}\text { AB } \\
0.9\end{array}$ & \\
\hline Characteristics & \multicolumn{8}{|c|}{ Avr. cluster weight (g.) } & \multicolumn{8}{|c|}{ Shot berries \% } \\
\hline b1 66 eyes/vine & 350.0 & 359.0 & 370.0 & 359.7 & 341.9 & 353.0 & 364.0 & 353.0 & 4.1 & 4.8 & 5.9 & 4.9 & 3.9 & 5.1 & 5.5 & 4.8 \\
\hline b2 72 eyes/vine & 361.0 & 371.0 & 381.0 & 371.0 & 353.0 & 365.0 & 375.0 & 364.3 & 4.9 & 5.9 & 7.1 & 6.0 & 4.7 & 6.2 & 7.2 & 6.0 \\
\hline b3 78 eyes/vine & 372.0 & 383.0 & 394.0 & 383.0 & 363.0 & 374.0 & 384.0 & 373.7 & 5.6 & 6.7 & 7.9 & 6.7 & 5.5 & 6.9 & 8.0 & 6.8 \\
\hline b4 84 eyes/vine & 383.0 & 399.0 & 410.0 & 397.3 & 374.0 & 389.0 & 396.0 & 386.3 & 6.9 & 8.0 & 8.9 & 7.9 & 7.1 & 8.1 & 8.9 & 8.0 \\
\hline Mean (A) & 366.5 & 378.0 & 388.8 & & 358.0 & 370.3 & 379.8 & & 5.4 & 6.4 & 7.5 & & 5.3 & 6.6 & 7.4 & \\
\hline New L.S.D at 5\% & $\begin{array}{c}\text { A } \\
9.0\end{array}$ & $\begin{array}{c}\text { B } \\
10.0\end{array}$ & $\begin{array}{c}\text { AB } \\
17.3\end{array}$ & & $\begin{array}{c}\mathrm{A} \\
9.2\end{array}$ & $\begin{array}{c}\text { B } \\
10.0\end{array}$ & $\begin{array}{c}\mathbf{A B} \\
17.3\end{array}$ & & $\begin{array}{c}\text { A } \\
0.4\end{array}$ & $\begin{array}{c}\text { B } \\
0.4\end{array}$ & $\begin{array}{c}\text { AB } \\
0.7\end{array}$ & & $\begin{array}{c}\text { A } \\
0.4\end{array}$ & $\begin{array}{c}\text { B } \\
0.4\end{array}$ & $\begin{array}{l}\text { AB } \\
0.7\end{array}$ & \\
\hline
\end{tabular}

Fayoum J. Agric. Res. \& Dev., Vol. 31, No.2, July, 2017 


\section{Percentage of shot berries:}

It is evident from the date in Table (3) that delaying dates of pruning from $24^{\text {th }}$ Dec. to $7^{\text {th }}$ Jan. as well as increasing vine loads from 66 to 84 eyes/vine and lengths of fruiting canes from 9 to 12 eyes per each significantly caused a gradual increase on the percentage of shot berries. The lowest values $(4.1 \& 3.9 \%)$ were recorded on the vines that pruned on $24^{\text {th }}$ Dec. leaving 66 eyes/vine. These results were true during both seasons.

\section{Quality of the berries:}

It is noticed from the data in Table (4) that delaying dates of pruning significantly was followed by a gradual reduction on berry weight and total acidity $\%$ and promotion on T.S.S.\%, and reducing sugars\%. A significant reduction were observed on berry weight and total acidity $\%$ and promotion on T.S.S. $\%$ and reducing sugars $\%$ with increasing levels of vine load from 66 to 84 eyes and fruiting cane length from 9 to 12 eyes per each. One can state that the best results with regard to chemical characters were obtained due to pruning on $7^{\text {th }}$ Jan. leaving 84 eyes/vine. Similar results were announced during both seasons.

Table (4): Effect of different dates of pruning and/or vine load levels on berry weight and percentages of T.S.S, total acidity and reducing sugars of Superior grapevines during 2011/2012 and 2012/2013 seasons.

\begin{tabular}{|c|c|c|c|c|c|c|c|c|c|c|c|c|c|c|c|c|}
\hline \multirow{4}{*}{$\begin{array}{l}\text { Vine load } \\
\text { levels ( B ) }\end{array}$} & \multicolumn{8}{|c|}{ Avr. Berry weight (g.) } & \multicolumn{8}{|c|}{ T.S.S.\% } \\
\hline & \multicolumn{4}{|c|}{ 2011/ 2012} & \multicolumn{4}{|c|}{$2012 / 2013$} & \multicolumn{4}{|c|}{ 2011/ 2012} & \multicolumn{4}{|c|}{$2012 / 2013$} \\
\hline & \multicolumn{16}{|c|}{ Dates of pruning (A) } \\
\hline & $\begin{array}{c}a_{1} \\
24 \\
\text { Dec. }\end{array}$ & $\begin{array}{c}\mathbf{a}_{\mathbf{2}} \\
\mathbf{3 1} \\
\text { Dec. }\end{array}$ & $\begin{array}{c}\mathbf{a}_{3} \\
7 \\
\text { Jan. }\end{array}$ & $\begin{array}{c}\text { Mean } \\
\text { (B ) }\end{array}$ & $\begin{array}{c}\mathbf{a}_{1} \\
\mathbf{2 4} \\
\text { Dec. }\end{array}$ & $\begin{array}{c}\mathbf{a}_{2} \\
\mathbf{3 1} \\
\text { Dec. }\end{array}$ & $\begin{array}{c}\mathbf{a}_{3} \\
7 \\
\text { Jan. }\end{array}$ & $\begin{array}{c}\text { Mean } \\
\text { (B ) }\end{array}$ & $\begin{array}{c}a_{1} \\
24 \\
\text { Dec. }\end{array}$ & $\begin{array}{c}\mathbf{a}_{2} \\
\mathbf{3 1} \\
\text { Dec. }\end{array}$ & $\begin{array}{c}\mathbf{a}_{3} \\
7 \\
\text { Jan. }\end{array}$ & $\begin{array}{c}\text { Mean } \\
\text { (B ) }\end{array}$ & $\begin{array}{c}a_{1} \\
24 \\
\text { Dec. }\end{array}$ & $\begin{array}{c}\mathbf{a}_{2} \\
31 \\
\text { Dec. }\end{array}$ & $\begin{array}{c}\mathbf{a}_{3} \\
7 \\
\text { Jan. }\end{array}$ & $\begin{array}{c}\text { Mean } \\
\text { (B ) }\end{array}$ \\
\hline b1 66 eyes/vine & 3.8 & 3.73 & 3.66 & 3.7 & 3.87 & 3.8 & 3.70 & 3.8 & 17.5 & 18 & 18.4 & 18.0 & 17.6 & 18.1 & 18.6 & 18.1 \\
\hline b2 72 eyes/vine & 3.74 & 3.66 & 3.59 & 3.7 & 3.79 & 3.69 & 3.60 & 3.7 & 17.8 & 18.4 & 18.9 & 18.4 & 17.9 & 18.7 & 19.2 & 18.6 \\
\hline b3 78 eyes/vine & 3.65 & 3.59 & 3.52 & 3.6 & 3.7 & 3.59 & 3.51 & 3.6 & 18.2 & 19 & 19.4 & 18.9 & 18.4 & 19.3 & 20 & 19.2 \\
\hline b4 84 eyes/vine & 3.57 & 3.51 & 3.44 & 3.5 & 3.59 & 3.51 & 3.44 & 3.5 & 18.6 & 19.5 & 19.9 & 19.3 & 18.9 & 19.9 & 20.4 & 19.7 \\
\hline Mean (A) & 3.7 & 3.6 & 3.6 & & 3.7 & 3.6 & 3.6 & & 18.0 & 18.7 & 19.2 & & 18.2 & 19.0 & 19.6 & \\
\hline New L.S.D at 5\% & $\begin{array}{c}\mathrm{A} \\
0.06\end{array}$ & $\begin{array}{c}\text { B } \\
\mathbf{0 . 0 7}\end{array}$ & $\begin{array}{c}\text { AB } \\
0.12\end{array}$ & & $\begin{array}{c}\mathrm{A} \\
0.06\end{array}$ & $\begin{array}{c}\text { B } \\
0.07\end{array}$ & $\begin{array}{l}\mathrm{AB} \\
0.12\end{array}$ & & $\begin{array}{c}\text { A } \\
0.3\end{array}$ & $\begin{array}{c}\text { B } \\
0.3\end{array}$ & $\begin{array}{l}\text { AB } \\
0.5\end{array}$ & & $\begin{array}{c}\mathrm{A} \\
0.2\end{array}$ & $\begin{array}{c}\text { B } \\
\mathbf{0 . 3}\end{array}$ & $\begin{array}{l}\text { AB } \\
0.5\end{array}$ & \\
\hline Characteristics & \multicolumn{8}{|c|}{ Total acidity \% } & \multicolumn{8}{|c|}{ Reducing sugars \% } \\
\hline b1 66 eyes/vine & 0.699 & 0.675 & 0.650 & 0.675 & 0.705 & 0.679 & 0.659 & 0.681 & 15.7 & 16.0 & 16.5 & 16.1 & 15.9 & 16.6 & 17 & 16.5 \\
\hline b2 72 eyes/vine & 0.68 & 0.655 & 0.620 & 0.652 & 0.680 & 0.658 & 0.63 & 0.656 & 16.1 & 16.6 & 17.0 & 16.6 & 16.4 & 17.1 & 17.5 & 17.0 \\
\hline b3 78 eyes/vine & 0.659 & 0.622 & 0.601 & 0.627 & 0.660 & 0.631 & 0.605 & 0.632 & 16.6 & 17.1 & 17.6 & 17.1 & 16.8 & 17.4 & 17.8 & 17.3 \\
\hline b4 84 eyes/vine & 0.63 & 0.610 & 0.589 & 0.610 & 0.630 & 0.640 & 0.594 & 0.621 & 17.2 & 17.7 & 18.2 & 17.7 & 17.2 & 17.7 & 18.1 & 17.7 \\
\hline Mean (A) & 0.667 & 0.641 & 0.615 & & 0.669 & 0.652 & 0.622 & & 16.4 & 16.9 & 17.3 & & 16.6 & 17.2 & 17.6 & \\
\hline New L.S.D at $5 \%$ & \begin{tabular}{|c|} 
A \\
0.014
\end{tabular} & \begin{tabular}{|c|} 
B \\
0.015
\end{tabular} & $\begin{array}{c}\text { AB } \\
0.026\end{array}$ & & $\begin{array}{c}A \\
0.015\end{array}$ & $\begin{array}{c}\text { B } \\
0.015\end{array}$ & $\begin{array}{c}\text { AB } \\
0.026\end{array}$ & & $\begin{array}{c}A \\
0.3\end{array}$ & $\begin{array}{c}\text { B } \\
0.3\end{array}$ & $\begin{array}{l}\text { AB } \\
0.5\end{array}$ & & $\begin{array}{c}\mathrm{A} \\
0.3\end{array}$ & $\begin{array}{c}\text { B } \\
0.3\end{array}$ & $\begin{array}{c}\text { AB } \\
0.5\end{array}$ & \\
\hline
\end{tabular}

\section{DISCUSSION}

The beneficial effects of adjusting date of pruning as well as vine load levels and lengths of fruiting canes on controlling growth and yield of the vines could explain the present results. Percentage of fruiting buds, growth magnitude, vine nutritional status and the ratio between growth and fruiting state greatly governed by adjusting dates of pruning and vine loads during winter pruning.

Fayoum J. Agric. Res. \& Dev., Vol. 31, No.2, July, 2017 
Faissal F. Ahmed"; et al.,

These results are in harmony with those obtained by Al-Khayat and Al-

Dujaili, (2001), Attia, (1998); Avenant, (1998); Ahmed-Ansam, (2002); Jarad, (2004);Hussein, (2009); Fawzi et al., (2010); Abdel- Mohsen, (2013); Abu- Zinada, (2015); Aly et al., (2015); Rahmani et al., (2015) and Khalaf, (2017).

\section{CONCLUSION}

Leaving 84 eyes/vine ( 6 fruiting canes x 12 eyes +6 renewal spurs $\mathrm{x}$ two eyes) on $7^{\text {th }}$ Jan. gave the best results with regard to yield and chemical characteristics of Superior grapevines grown under Fayoum environmental conditions.

\section{REFERENCES}

Abdel-Mohsen, M.A. (2013): Application of various pruning treatments for improving productivity and fruit quality of Crimson Seedless grapevine. World Journal of Agricultural Sciences 9 (5): 377-382.

Ahmed- Ansam, S.A., (2002). Effect of cane length on bud behaviour, growth and productivity of "Superior" grapevines. M.Sc. Thesis, Fac. of Agric. Cairo Univ.

Abu-Zinada, I.A. (2015): Effect of GA3, girdling or pruning on yield and quality of 'Pereletta' Seedless Grape. American Journal of Agriculture and Forestry 3 (5), 230-233.

Akopyan, G. A. and Khasapetyan, R. T., ( 1994 ). Optimizing bud load and pruning length of fruiting canes. Sadovodstvo i Vinogradarstvo ( 3): 12.

Al-Khayat, R.A. and Al-Dujaili, J.A. ( 2001 ). Effect of pruning and nitrogen fertilizer levels and their interaction on quantitive characteristics of two grape cultivars (Halwani and Kamali) (Vitis Vinifera, L.). Dirasat Agric. Sci., 28(1):87:98.

Ahmed, F. F. and Morsy, M. H. (1999): A new method for measuring leaf area in different fruit species. Minia J. Agric Res. \& Develop. Vol. (19) pp 97-105.

Aly M. A.; Ezz Thanaa, M.; Awad Rehab M. and Abou-Elmaaty, A. M. ( 2015 ). Effect of different plastic sheet coverings and pruning time on yield and yield components of table grape cv. Superior. Hort. Sci. J. of Suez Canal Univ. No (1): 1-14.

Association of Official Agricultural Chemists (2000): Official methods of Analysis (A.O.A.C), $17^{\text {th }}$ Ed. Benjamin Franklin Station, Washington D.C. U.S.A. pp.490-510.

Attia, M.F.S. ( 1998 ). Effect of foliar nutrition, different vine bud loads and spur length on some vegetative and fruiting characters of Red Roomy grapevines. M. Sc. Thesis Fac. of Agric. Minia Univ. Egypt.

Fayoum J. Agric. Res. \& Dev., Vol. 31, No.2, July, 2017 
BEHAVIOUR OF SUPERIOR GRAPEVINES TO.

Avenant, J.H. ( 1998 ). The effect of pruning level on the performance of Festival seedless. Deciduous Fruit Grower, 48:5, 71-3 (C.F. Hort. Abst., 68 (10):8436).

Bouard, J. ( 1966 ). Recherches physiologiques sur et en particulier sur laoutment des serments. Thesis Sci. Nat.Bardeux, France p.34.

Chapman, K.R. (1990): Table grapes in the Tropics Areas for Research and Market Apportunities. Acta Horticulture: III International Workshop on Temperate Zone Fruits in the Tropics and Subtropics 279: 910,96.

Fawzi, M.I.F.; Shahin, M.F.M. and Kandil, E.A. (2010). Effect of bud load on bud behavior, yield, cluster characteristics and some biochemical contents of the can of Crimson seedless grapevines. Journal of American Science 6(12).

Hussein, M.A. (2009): Adjusting the suitable vine bud load as well as the optimum date and concentration of Dormex for advancing bud burst and improving productivity of Superior grapevines. Ph.D. Thesis Fac. of Agric. Minia Univ. Egypt.

Jarad, A. (2004): Effect of fruit canes and eyes had load on the productivity of a local grape variety under the conditions of Deir El- Zor city. Bull Fac. Agric. Cairo Univ, 55: 603-608.

Khalaf, M.A.M. (2017): Effect of fruiting spur length and spraying seaweed extract on yield and berries quality of Early sweet grapevines. M.Sc. Thesis Fac. of Agric. Minia Univ. Egypt.

Lane, J. H. and Eynon, L. (1965): Determination of reducing sugars by means of Fehling's solution with methylene blue asindicatorA.O.AC. Washington D.C., U.S.A. pp. 490 - 510.

Mead, R.; Currnow, R. N. and Harted, A. M. (1993): Statistical Methods in Agricultural and Experimental Biology. $2^{\text {nd }}$ Ed. Chapman and Hall, London pp. 10- 44.

Rahmani, M.; Bakhshi, D. and Qolov, M. ( 2015 ). Impact of pruning severity and training systems on red and white seedless table grape ('Vitis vinifera') . Australian Journal of Crop Science, Vol. 9, No. 1: 55-61.

Summer, M.E. (1985): Diagnosis and Recommendation Integrated system (DRIS) as a guide to orchard fertilization. Hort. Abst. 55(8): 7502.

Von-Wettstein, D.V. ( 1957 ). Chlorophyll latele ubder submikro skopiche formwechsel der plastids. Experimental cell Res. 12: 427-433.

Wilde, S. A.; Corey, R. B.; Layer, J. G. and Voigt, G. K. (1985): Soils and Plant Analysis for Tree Culture. 3rd Ed. Oxford and IBH publishing Co., New Delhi, India. pp. 529 - 546.

Fayoum J. Agric. Res. \& Dev., Vol. 31, No.2, July, 2017 


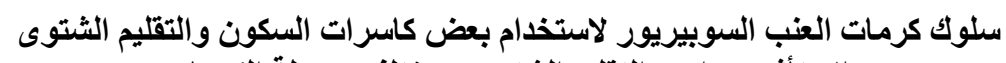

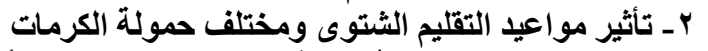

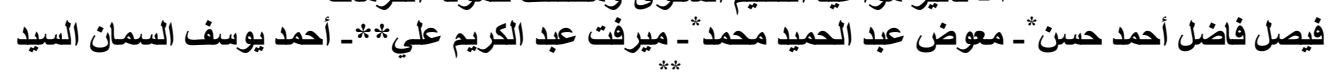

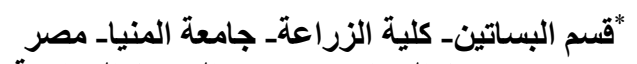

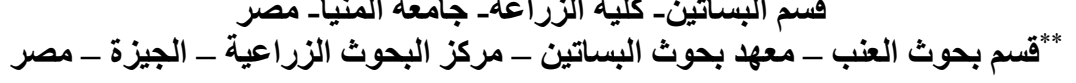

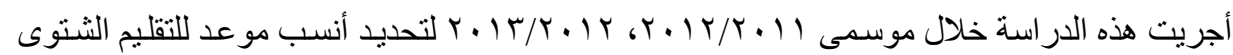

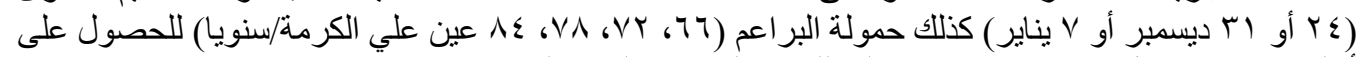

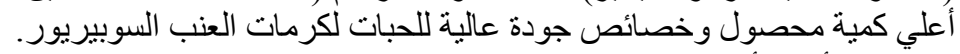

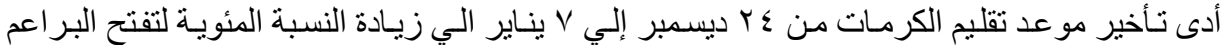

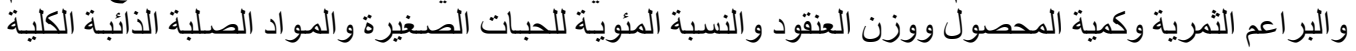

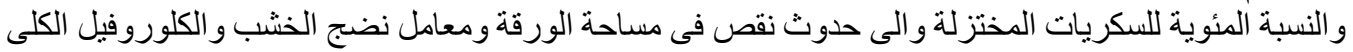

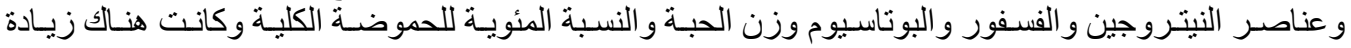

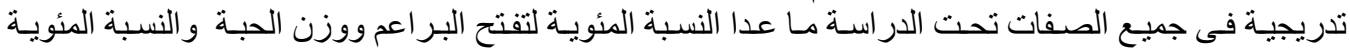

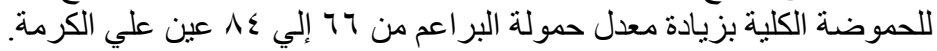

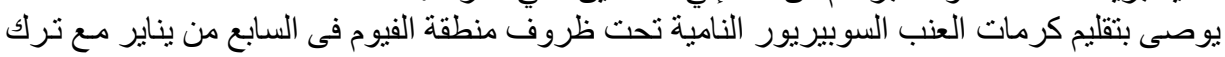

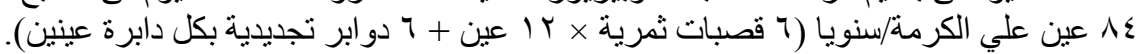

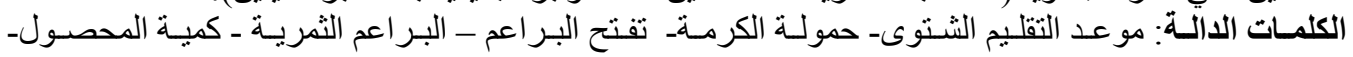

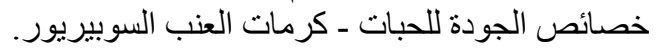

Fayoum J. Agric. Res. \& Dev., Vol. 31, No.2, July, 2017 